\title{
A Study on User Participation Based Outdoor \& Indoor Spatial Information Building
}

\author{
Yongwon $\mathrm{Cho}^{1 *}$, Jinwon $\mathrm{Choi}^{2}$ \\ ${ }^{1}$ Researcher Engineer, Virtual Builders Co., Ltd., Seoul, Korea. (IACSIT Member 80348498). \\ ${ }^{2}$ CEO, Virtual Builders Co., Ltd., Seoul, Korea. \\ * Corresponding author. Tel.: (82)10.9470.1010; email: avenuel88@gmail.com \\ Manuscript submitted June 3, 2014; accepted August 25, 2014. \\ doi: 10.17706/ijcce.2015.v4.392
}

\begin{abstract}
Recently, outdoor \& indoor spatial information technology closer to reality 3D space. So spatial information services market is growing now. A variety of information services and web-based content, to provide information services through the space. In addition, consumers make to direct spatial information service more than just a service provider. That means satisfy the needs of the consumer now. We have a lot of high quality smart devices. So, we will available to upload or share of web.

In order to use the high quality image upload large amounts of processing is required. This study is outdoor \& indoor map building based on the 3D data. And we will make to management for integrated platform. We have to platform project name is 'CRETA'.
\end{abstract}

Key words: Big Data, crowd mapping, CRETA platform, user participation, spatial information service.

\section{Introduction}

Buildings are getting bigger and more complex. The spread of smart devices, a wide range of services as a new chapter in the fusion of interior space is increasingly emerging as important. And, according to a report by the U.S. Environmental Protection Agency said, "For about $80 \%$ on average over the entire life takes place in the room. Moreover, there is a need of increasing indoor activities. Also disaster prevention against terrorism and accidents is an important issue that needs to be resolved.

In the field of spatial information process of building a real time digital map of the various types of information that must be collected. That service is spatial information in the field of outdoor and indoor space together without distinction to handle demand. Recently, spatial information technology service deployment from real 3D space, multi-dimensional space based information service and web-based content through a variety of service.

We are looking for the convenience of living a variety of techniques have been developed to be used in real life. This made possible the reality is that smart devices. Smart phone applications, instead of finding themselves on the existing can be. Typical examples, directions or ordering food, data search, contact search, public transport, such as arrival time of the service through the smart devices can be used anywhere, anytime. Percentage of smart phone users worldwide in 2012 and the end of the 1 billion people use around the world, given the population of 7 billion people 1 out of 7 people are using the smart devices.

Global companies such as Google, Apple and Microsoft have been constructing indoor spatial information. They have been offering 2D indoor map and pictures (360ํㅡㄹ panorama) based indoor map provision. Now 
they are expanding the construction for indoor map from the U.S to worldwide. These services are predicted about 120,000 places in 2016.

In this study, many LBS services can be provided based on indoor spatial information. With the rapid development in hardware and software of wireless communications, applications of real-time positioning systems have been widely used; among them the GPS is the most popular. Creating 3D indoor maps is getting even more useful by providing maps for indoor spatial information. People needs for LBS services for their safety and convenience through the integration of indoor spatial information.

\section{Technical Trends}

There are at least 170 companies today working on indoor location, indoor maps, building tracking and indoor navigation add to outdoor position. The growth of this market has been impressive over the last 10 years. Indoor is the last mile and the next frontier for the location services market. Indoor maps emerged rapidly in 2009 as started up such as Point Inside, Micello and Nokia. Released their indoor content, while indoor mapping data is available for indoor navigation applications, associated location determination is still a challenge. A number of tagging \& infrastructure based technologies can deliver to precise indoor location, but it is tough to make these solutions ubiquitous.

Image data representative of the services offered by the MS of the 'Photosynth'. 'PhotoSynth' in the case of individuals who hold multiple pictures, similar to the actual appearance by placing shall automatically complete three-dimensional picture (Fig. 1). Three-dimensional pictures on-line store, and the location of the photos on the map by inserting has been able to show to other users.

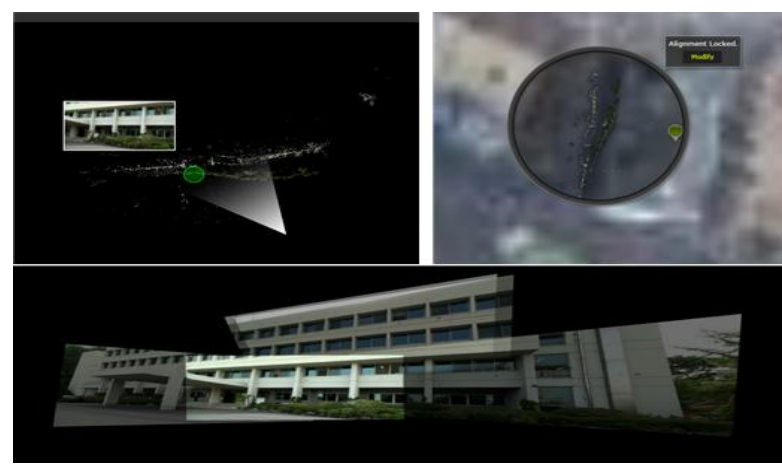

Fig. 1. 'Photosynth' 3D point cloud and inserted on the map (above), 3D photos (below).

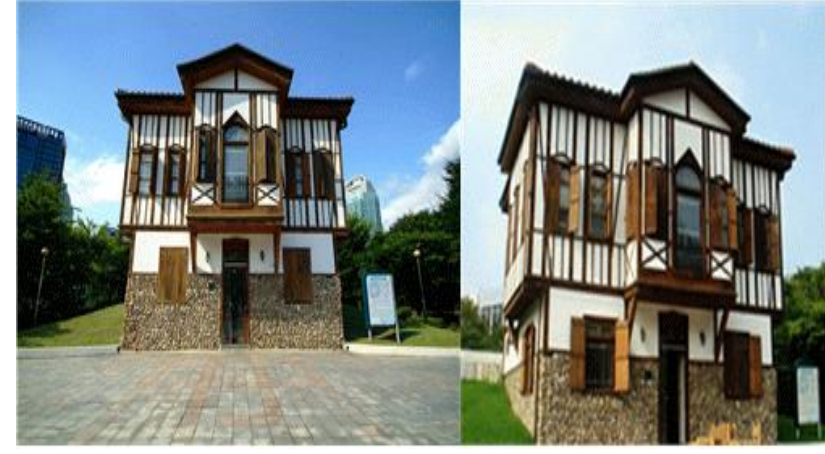

Fig. 2. Take a Photo at 'Ankara House'Seoul, Korea (Yongwon Cho).

Multi-dimensional real-spatial information (Hyper-Live Map) to in the image data obtained through the Point Cloud to 3D Reconstruction purpose of the study, performed experiments by CCTV.

Experimental results establish the structure only when recording CCTV, when shooting closed areas or shaded areas 50 percent due to the building was possible, To compensate for this, the video camera, or smart phone video by adding the results of building CCTV video was more effective than just that building. Fig. 3 shows the Konkuk University main building with CCTV video camera image is using that constructed.

\section{High Quality Image Data Acquisition Methods Research}

In the field of spatial information process of building a real time digital map of the various types of information that must be collected. That service is spatial information in the field of indoor and outdoor space together without distinction to handle demand [1]. Recently, spatial information technology service deployment from real 3D space, multi-dimensional space based information service and web-based content through a varieties of services [2]. 
South Korea government makes the 3D map by 'V-world'. 'V-world' seems like 'Google Earth'. And 'Naver' and 'Daum' map service in the aerial view, real photo view or normal view [1].

We are looking for the convenience of living a variety of techniques have been developed to be used in real life. This made possible the reality is that smart devices. Smart phone applications, instead of finding themselves on the existing can be. Typical examples, directions or ordering food, data search, contact search, public transport, such as arrival time of the service through the smart devices can be used anywhere, anytime. Percentage of smart phone users worldwide in 2012 and the end of the 1 billion people use around the world, given the population of 7 billion people 1 out of 7 people are using the smart devices [3]. This spread is the spread of smart devices, a wide range of services as a new chapter in the fusion of interior space is increasingly emerging as important. And, according to a report by the U.S. Environmental Protection Agency said, "For about 80\% on average over the entire life takes place in the room" [4].

To mention above can be obtained through the high quality image data. So high quality image data in other ways to compensate people carrying smart phones and digital cameras of through user-participation. Smart phones and digital cameras very portable, and technology to the development of high quality images can be obtained easily [5]. General with the consent of the user to upload images can be obtained through an internet search [6]. When these images are acquired to manage large amounts of images to solve this problem, which is currently active in the big data and cloud computing to take advantage of the one that you think is an alternative.

This study is collected to camera user pictures at any position, so we will make to high quality 3D map data. That collected to smart camera were taking pictures (Fig. 2).

In many parts of panorama pictures taken from any locations to 3D modeling was made it. That is possible based on these 3D modeling of user participation shown (Fig. 3).

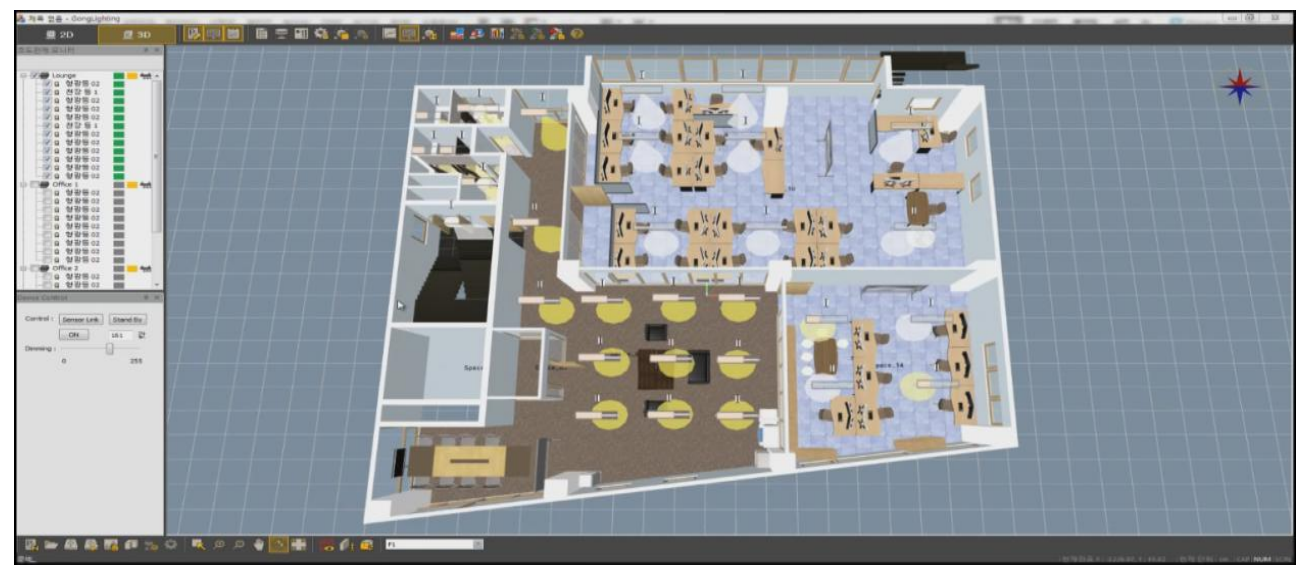

Fig. 3. Handling to program 'GongBuilder' at 'Ankara House' Seoul, Korea.

\section{Fusion of High Quality Image Data to Suggestion}

\subsection{Big Data}

Recently, GPS-equipped smart phone due to the prevalence of SNS activation, social media, due to the growth of diverse and numerous amounts of text, video, location data is generated in real-time position information, as well as the behavior of people and ideas and through SNS comments are able to analyze and predict (Fig. 4). Most of this data collection, storage, retrieval, analysis, visualization, and difficult to non/semi-structured data, if not used properly useless, that can be data [7]. Big Data and efficient processing of such data, analysis, and in order to take advantage of was the emergence, Big Data is usually data volume, variety, velocity as a combination of three factors is characterized by change [8]. Big Data and analysis techniques for processing such data, the text mining, opinion mining, social network analysis, 
cluster analysis has dual images similar to nested characteristics of the object together with the cluster analysis technique was used for outgoing [9].

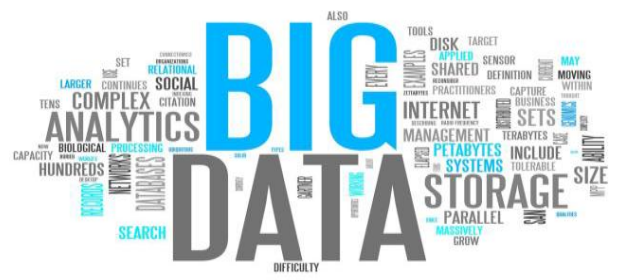

Fig. 4. Big data (Fotolia).

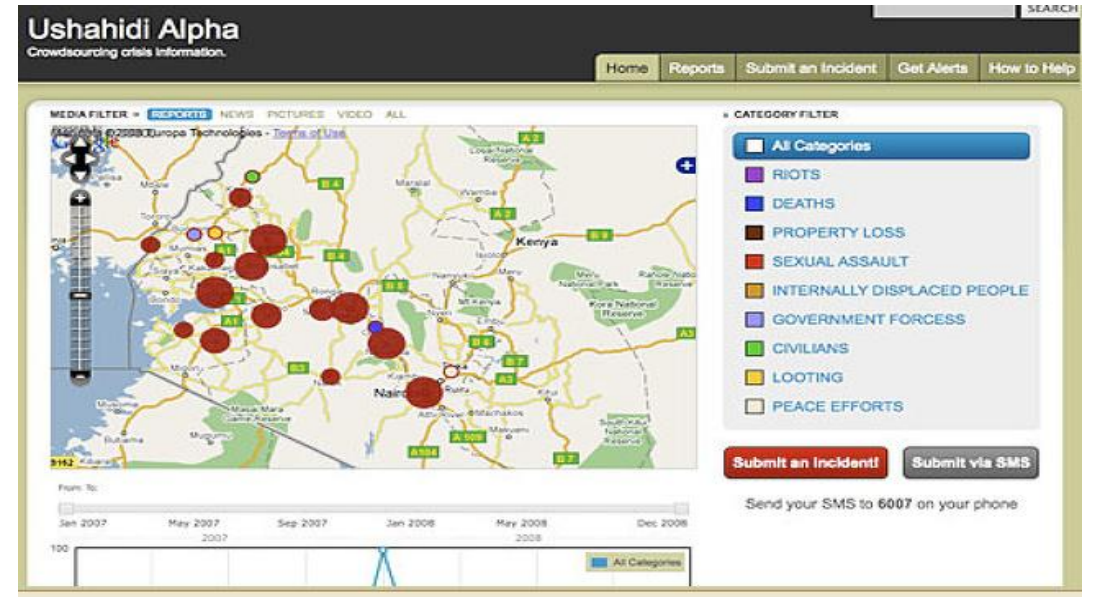

Fig. 5. Crowd mapping (Ushahidi).

\subsection{Crowd Mapping}

When As mentioned earlier, many of Crowdsourcing development and through user participation can be consumed. Today based on the evolution of online communication technologies with the public to show the infinite possibilities [10]. Crowdsourcing is therefore beneficial to both businesses and the public to be used, a systematic procedure based on a clear sense of purpose can be satisfied through the participants should be provided with appropriate incentives [11].

As said, Crowd mapping is designed and built by the team behind Ushahidi, a platform that was originally built to crowd source crisis information. As the platform has evolved, so have its uses. Crowd mapping now allows users to set up their own deployments of Ushahidi without having to install it on a web server. Since its release in 2010, prominent deployment of Crowd mapping have documented the global 'occupy' protests and the 2011 London anti-cuts protest [12].

On 31 December 2010, the Ushahidi team announced Crowd mapping: Check in, a geo-social add on to Crowd mapping that allows users to create a white label alternative to sites like Foursquare and Gowalla [4] [12]. Rather than filling out submission forms online, check in allow Crowd mapping users to expedite data entry to their deployment, focus first on location and adding more detailed information later [13]. Ushahidi describes the effort as 'check in with a purpose' (Fig. 5).

\subsection{User Participation}

Wikipedia users to make their way directly participated map programs are gaining popularity recently. Focusing on user participation in Wikipedia map Global Positioning System (GPS)-equipped people to use a smartphone without any prior knowledge help us be able to create a map of the world. Participation 
typically made of an open-source map 'Waze' and Open Street Map there (Fig. 6).

Israel Tal Aviv 'Waze' for the first time in 2006, began. Users are not marked on the map into a dead end when the Wise stood no way connected to the place on the map to display the next place to visit for people who can help. 'Waze' is now the driver of the 14 million people worldwide and is used to edit the map and 45,000 people living in 5,000 people in his area manager to verify the accuracy of the map is active.

Open Street Map is a 'Waze' was born with a similar purpose or non-profit model is more like Wikipedia. Open Street Map is like Wikipedia, which anyone can add information to connect to the home page and can be modified because it is free to use. Usage of open street map homepage and searching for the place is similar to Google Maps. However, unlike Google Maps and Open Street Map is a map, not just anyone can use without having to pay a geographic information features.

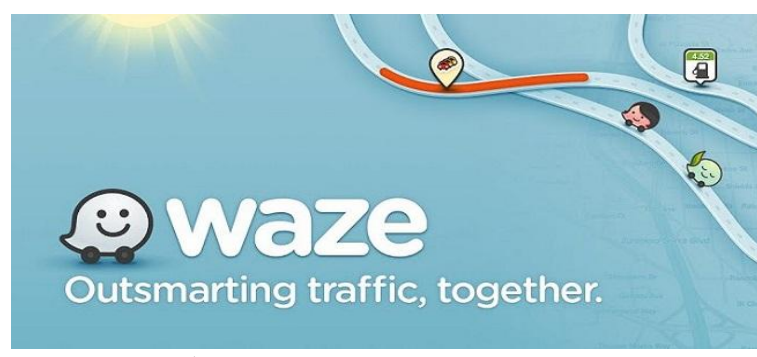

Fig. 6. 'Waze' logo (www.waze.com).

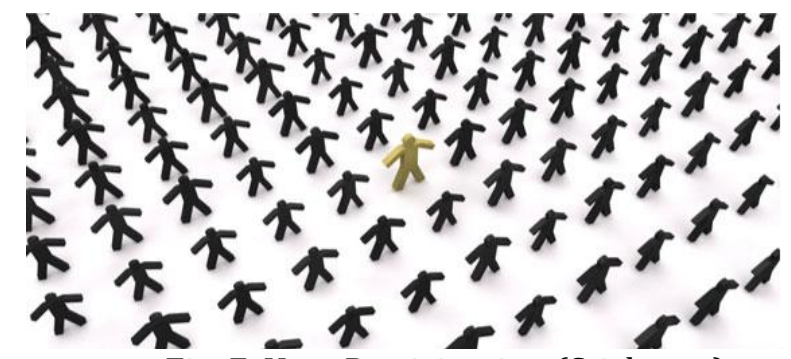

Fig. 7. User Participation (Stickmen).

Recent popular location-based social network services company Foursquare have the same characteristics of the open street map to identify the user where their friends are staying open, make sure that you had to use a street map unveiled last month.

Disaster relief organizations are also 'maps of the terrain is changed just change' increasingly rely on participatory map. Earthquake in 2010 significantly changed the topography of the entire country rescuers in Haiti is that using the example of the open street map. Nine trillion won at the time of the Haiti relief efforts using real-time information to modify the map where the terrain is changed when it arrived and prayed to inform the open street map (Fig. 7).

Jonathan Bennett, open street map users "that there is no other way to create a map from the open street map is not inferior to the rate of progress," he said [14].

\section{Development to 'CRETA' Platform}

In this study, user participation to outdoor \& indoor build to 3D map(Fig. 8). By default, the collected to data, make to 3D modeling experimental.

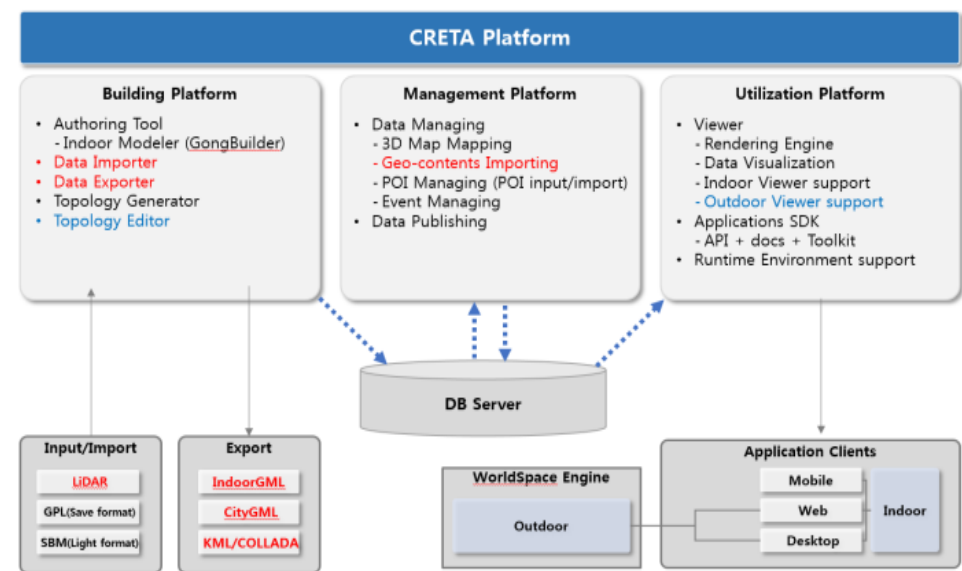

Fig. 8. Indoor \& Outdoor integrate platform (CRETA). 


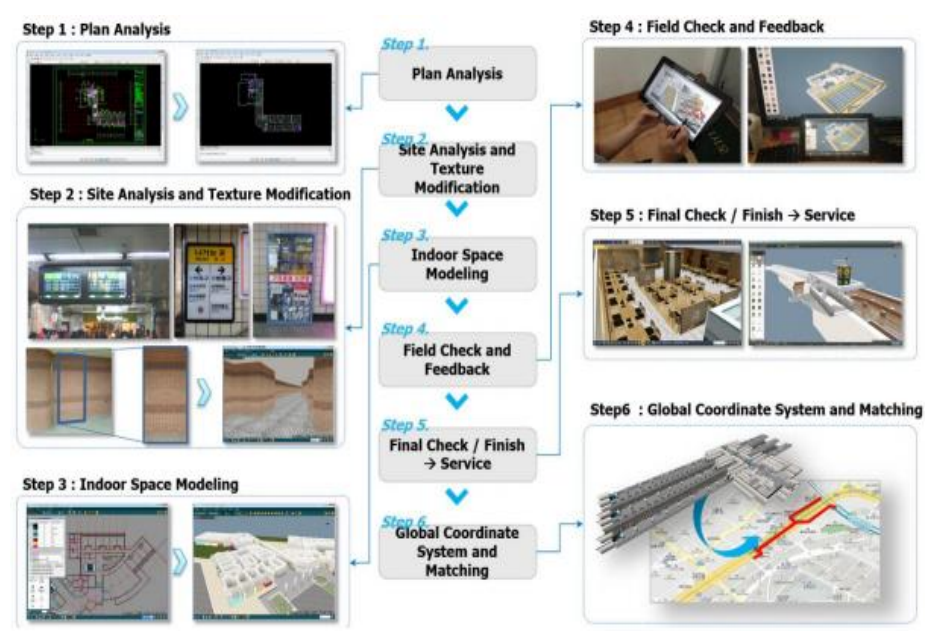

Fig. 9. The map of construction Process.

There are three technologies to construct 3D outdoor \& indoor map mainly. The technologies are first, 3D laser scanning or high quality image are useful for space. We can construct outdoor \& indoor map utilizing scan data. Then we can acquire 3D data and purify it and eliminate noise. Second, we can generate polygons with refined data and cover textures of actual images.

Second, Camera can shoot outdoor \& indoor changing angle or position freely. To construct outdoor \& indoor map, we can utilize that. We can shoot areas of the sphere using camera. Then we can estimate positions of points and models. We can cover textures of actual images in models and revise them.

Third, it is to implement in 3D based on completed drawing information about the outdoor \& indoor of the building existing in the basement or ground. It is useful technique to implement 3D indoor map of existing building that was built based on 3D modeling. It is can be used to create 3D outdoor \& indoor map in the future.

\section{Outdoor \& Indoor Spatial Information Data Construction}

The proposed method for creating 3D outdoor \& indoor map in this paper is based on the 'GongBuilder' and 'CRETA platform'. Which is the outdoor \& indoor spatial information modeling technology developed by 'Virtual Builders Co., Ltd.. The detailed construction process proposed in this paper is as shown (Fig. 3). The whole process is plan analysis (Step 1), site analysis and texture modification (Step 2), outdoor \& indoor modeling (Step 3), field check and feedback (Step 4), final check and finish (Step 5), and global coordinate system and matching (Step 6) (Fig. 9).

Step 1 analyzes completed drawings to enhance the understanding of space. The more target building a modeler understands, the better 3D outdoor \& indoor modeling will be conducted. Also, each floor uses the same reference coordinate in order to carry out 3D outdoor \& indoor modeling. The details are as below,

1) Review for floor plan, elevation, and sectional view of the target building

2) Drawing arrangement in order to reduce a file size

3) Calibration of reference coordinate system for each floor.

Step 2 investigates interior components that are not provided in 'GongBuilder' library through field survey and analysis. In order to depict similarly the indoor space model by using 'GongBuilder' tool, field investigation measures the size of components such as signboards, facilities and so on. After that, pictures of the checked components are taken in field. Textures for outdoor \& indoor components in the target space are produced by editing the pictures.

Step 3 target buildings or spaces are modeled through 3D outdoor \& indoor modeling too called the 
'GongBuilder' investigation by using 'GongBuilder' tool. Building elements such as space, wall, floor, roof, and etc. are formed, and exterior/interior facilities are disposed in the modeled space. To attribute like name and usage for each space are entered by user. All of the building elements are covered with the textures produced in the former step while adjusting the size of those.

Step 4 all spaces formed in the previous step are verified by an investigator in the field. The checker reports a modeling difference from the real space and improvements to the modeler through smart devices.

Step 5 finally examines and permits the end results of outdoor \& indoor spatial information model. If there is any problem, a manager is asked to revise the modeling errors.

It is possible to automatically generate a topology information outdoor \& indoor space. The data automatically generates a topology between the components of the building. It is also can automatically generate Zone/Connector/Zone-connector/Path standard OGC. It allows convert IndoorGML, which is an international standard format of outdoor \& indoor spatial information model. It also can convert KML, CityGML. CityGML is an international standard format of 3D outdoor \& indoor spatial information model (Fig. 10).

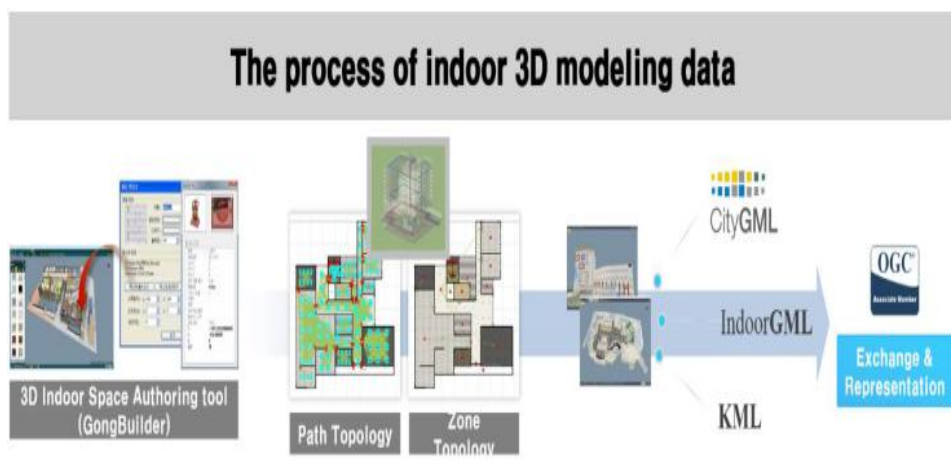

Fig. 10. Benefits of outdoor \& indoor 3D data.

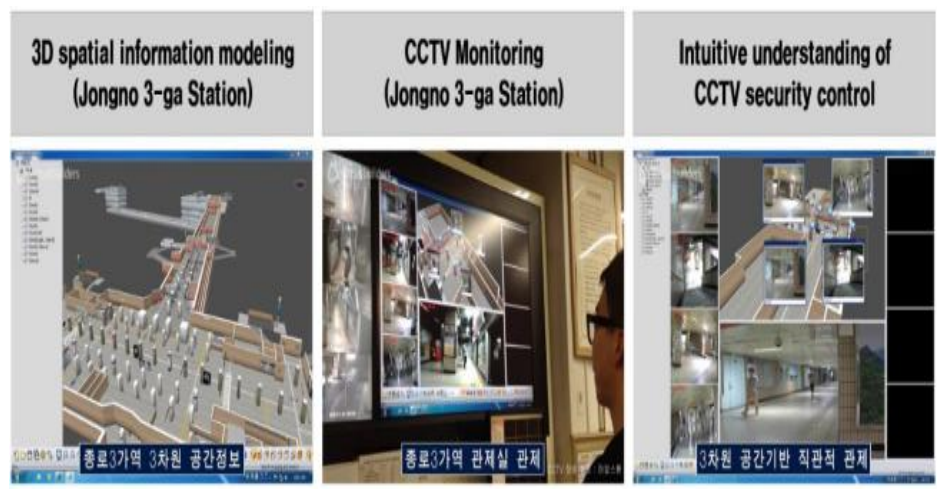

Fig. 11. Monitoring service based on Indoor 3D map.

Currently, 3D indoor modeling about main building in Seoul in progress (Fig. 11). Major public buildings, museums, art galleries, the main transfer station are targeted. Pilot service is operated and commercial service will start in coming year.

Physical security systems in conjunction with 3D indoor map is to monitor the security situation. Manager of huge spaces or buildings need intuitively monitor system. Currently, test service had operated in 'Jongno 3rd street Station', security monitoring service is developing advanced on the basis of the results of test service (Fig. 12).

The intermediate outcome of job creation project by information system planning bureau and 'Virtual Builders Co., Ltd.' was announced by major media. 


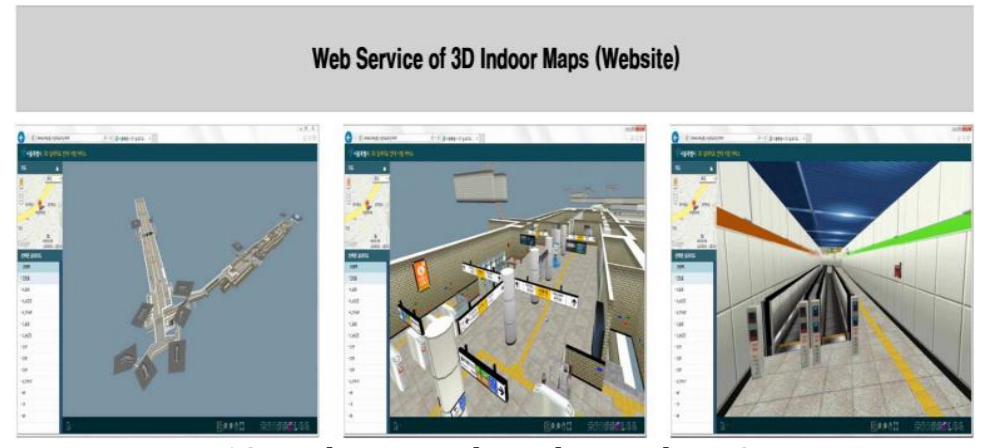

Fig. 12. Web service based on Indoor 3D map.

\section{Conclusion}

There are many future plans based on the 3D outdoor \& indoor maps. The invention system of indoor maps can be widely applied (Fig. 13).

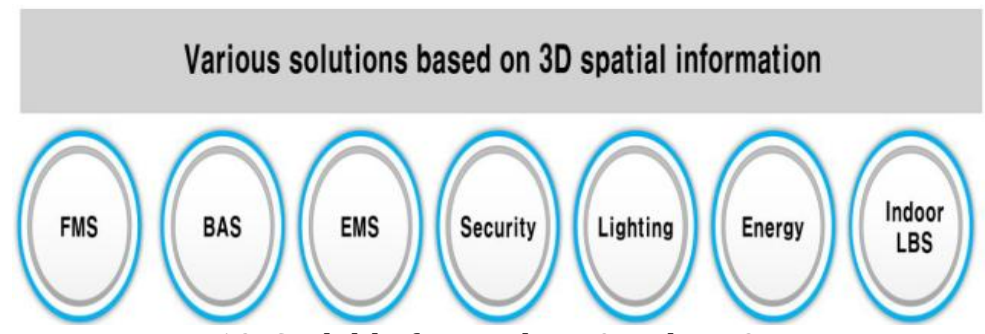

Fig. 13. Scalable for outdoor \& indoor 3D map.

This solution is being put to use in space management service. Managing space use and monitoring room status in Seoul. It provides to energy management service. It is possible to save energy through the energy monitoring system. Energy equipment layout and 3D visualization of energy consumption is visualized in each space in the building. It is provided to real time monitoring visualization, and it is possible to control system (Fig. 14).

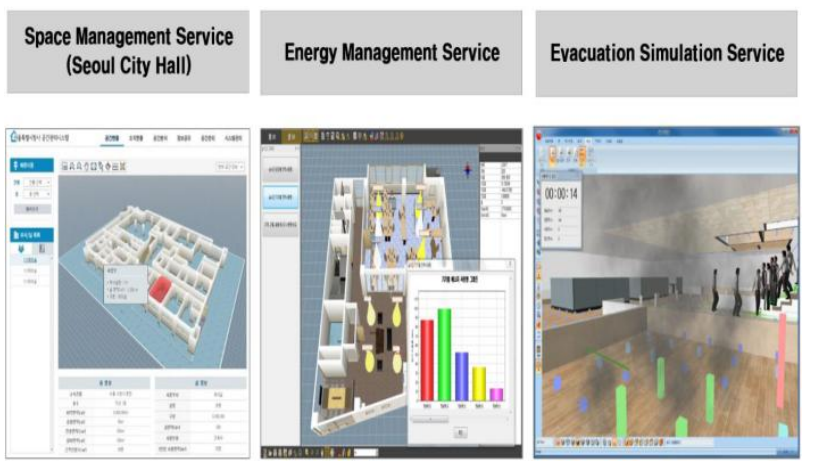

Fig. 14. Evacuation services based on 3D indoor map.

It is ready to provide to evacuation simulation service. This solution offered the application for fire and evacuation simulation and the prediction of route. It will be evacuation scenario expansion for disabled people to secure stability and evacuation simulation for high rise buildings, subway stations and large scale of building.

3D spatial information linked to various outdoor \& indoor services is required. These are available to be 
edited and modified. Moreover, the file of model can be saved as IndoorGML, CityGML and KML file. These files are viewed by the 'Viewer'. Outdoor \& indoor LBS is served with these principles. Also combine with mobile device and positioning system. We can provide outdoor \& indoor LBS (Fig. 15). Through mobile device, we provide virtual 3D space constructed by low polygon data. If we combine it with CRM data, we can use marketing service based on spatial information.

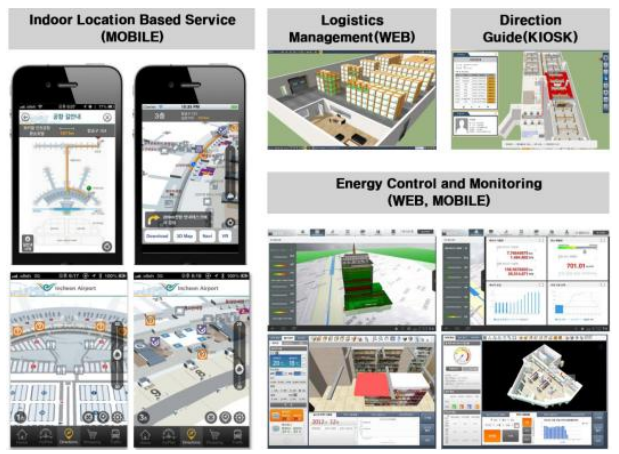

Fig. 15. LBS based on 3D indoor map.

3D spatial information based light energy integrated management solution is provided. Analyzing and controlling light energy, the system allows people to manage lights economically at building. The features are below. It is easy to handle the lighting maintenance control. It could be conserved by analyzing lighting consumption and lighting energy patterns in the spatial information. Also it would be maintained the efficient lighting system through a function of group management setting up the specific are of the whole building. And reduces time and cost through a function of integrated remote control based on the multi devices support of OSMU.

3D spatial information based CCTV security control integrated solution allows people to see real time data of building operation status interlocking with CCTVs and sensors at the inside of a building. The system monitors the status of CCTVs and sensors operation and control the security devices. The sensors layout simulation can be performed on 3D spatial information.

It provides floor layout scenes for each building levels with a level view option (Fig. 16). It provides intuitive scenes of the building space form view point using 7 kinds of view options. IT supports intuitive understanding of the building by linked 3D modeling to DVR server. Add CCTV channel after selecting the assigned DVR server. It monitors not only CCTV images, but also AVI files linked to POI. An added channel appears in a tree structure on the left side of the interface. CCTV POI can be assigned as desired. It displays real time CCTV image when CCTV POI is linked to DVR server. IT provides the location of CCTV when selected from the CCTV list in a tree structure on the left side of the interface.

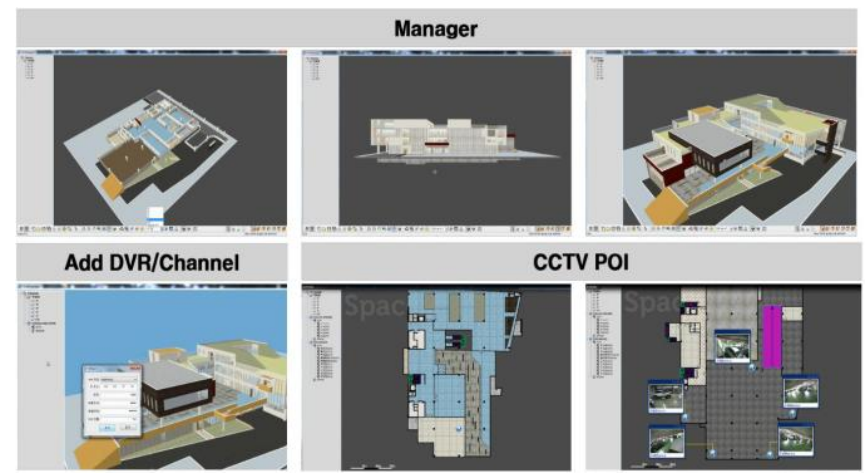

Fig. 16. CCTV monitoring service based on 3D indoor map. 
'Archi video' provides an intuitive and intelligent monitoring service by simulating spatial information. Optimum locations are decided while adjusting CCTV location on 3D virtual view. Virtual camera arrangement is all of set when POI is generated (Fig. 17).

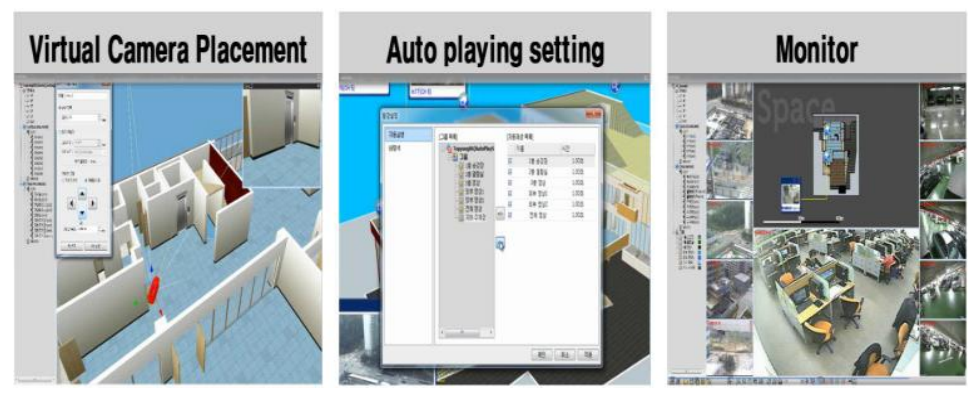

Fig. 17. Virtual camera for monitoring service.

Finally, 'CRETA' platform based 3D spatial information consists of space authoring (GongBuilder), space operation (GongManager) and space visualization (GongVue). It carries out more space values and new space experiences with realistic 3D technology. Our professional solutions at business, service and technology raise your values up through new perspective of space, challenge and integration with technology.

\section{Acknowledgment}

This work was supported by the National Research Foundation of Korea (NRF) grant funded by the Korea government (MEST) (No.2011-0028498)

\section{References}

[1] Lee, H. J. (2008). Using multi-dimensional spatial information orthophoto production plan realized. Journal of Korea Society Surveying, 26(3), 241-253.

[2] Lowe, D. G. (2004). Distinctive Image Features from Scale-invariant Keypoints.

[3] Nokia (2012). NOKIA Source Strategy Analytics.

[4] Ushahidi (2011). Checkins With a Purpose" Crisis-Mapping Platform Ushahidi Announces Crowdmap:CI. ReadWrite.

[5] Meesters, L., IJsselstejin, W. \& Seuntiens, P. (2003). Survey of perceptual quality issues in three-dimensional television system. In Proceedings of SPIE on stereoscopic Displays and Virtual Reality System $X, 5006$.

[6] Salton, G. (1989). Automatic Text Processing-the Transformation, Analysis and Retrieval of Information by Computer. Addison-Wesley Publishing Co..

[7] Cho, S. W. (2011). Big Data era technology. KT Institute of Technology (pp. 5-7). Korea.

[8] O'Reilly Radar Team (2012). Planning for Big Data. O'Reilly.

[9] Kang, M. M., Kim, S. R., Park, S. M. (2013). Analysis and utilization of big data. Journal of Information Science, Vol.30, 25-32.

[10] Yoo, J. H. (2010). The wisdom of the masses to be successful crowdsourcing is Mine. LG Business Insight, 46-53.

[11] Crowdsourcing Through Knowledge Marketplace (2010). SpinAct.

[12] Ushahidi (2011). Announcing checkins for Ushahidi and Crowdmap. Geo Spatial World.

[13] Ushahidi (2011). Launches Crowdmap Checkins. Geo Spatial World.

[14] Wikipedia Korea (2012). Hot Issue User Participation (Crowd Mapping) Map. Kyunghyang News. 


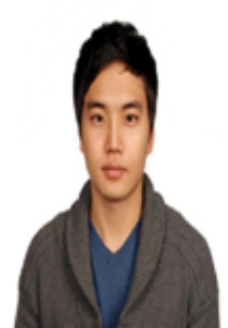

Yongwon Cho was born on 3rd August, 1988, Seoul, Republic of Korea and completed his B.Sc. degree in civil engineering from Suwon University of Engineering, Suwon, Republic of Korea in February 2012. He also completed his master degree in Department of Fusion Technology from Konkuk Graduate University, Seoul, Republic of Korea in February 2014. At present he is working at Virtual Builders Co., Ltd., Digital spatial lab. After graduation he research in indoor \& outdoor spatial information service. He always feels to do innovative research work that is reflected by a good number of his publications in international journals and conferences. His fields of interest are: Spatial Information, Image Matching, Indoor \& Outdoor Map, User Participation and GIS. (IACSIT member 80348498)

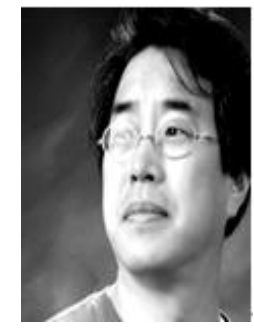

Jinwon Choi received his Ph.D degree in architecture from OHIO Graduate University, OHIO, United States; and now he is working at Virtual Builders corporation, CEO \& Founder. He was a university professor (Ajou \& Yonsei). He was development to 'PalladiOn', 'Gong Builder' and 'Abimo' tools. 'Abimo' is this year, new launching. Republic of Korea first made BIM modeling S/W. His ongoing research interests: BIM tools, GIS, LBS, Security and CCTV Solution. 